\title{
Addition of a Single Glycosylation Site to hAPN Blocks Human Coronavirus-229E Receptor Activity
}

\author{
DAVID E. WENTWORTH AND KATHRYN V. HOLMES \\ Department of Microbiology, University of Colorado Health Sciences Center, Denver, Colorado
}

\section{INTRODUCTION}

The cellular receptor for $\mathrm{HCoV}-229 \mathrm{E}$ is human aminopeptidase $\mathrm{N}$ (hAPN). Murine fibroblasts that are nonpermissive for $\mathrm{HCoV}-229 \mathrm{E}$ become susceptible after transfection with an hAPN expression plasmid (Yeager $e t$ al., 1992). In addition, antibodies to hAPN block infection of human neural cells by HCoV-229E (Lachance et al., 1998). hAPN, also called CD13, is a $150 \mathrm{kDa}$ glycoprotein that is a membrane peptidase (Look et al., 1989). APN is expressed by many cell types including epithelial cells of the kidney, intestine, respiratory tracts and at synaptic junctions in the CNS (Kenny and Maroux, 1982; Look et al., 1989; Riemann et al., 1999; Noren et al., 1997).

Aminopeptidase N (APN) also serves as the major receptor for serogroup I coronaviruses that infect pigs, cats and dogs (Delmas et al., 1992; Delmas et al., 1993; Tresnan et al., 1996). In general, APN is used in a species specific manner. However, feline APN (fAPN), serves as a receptor for many serogroup I coronaviruses including feline (FECoV and FIPV), porcine (TGEV), human (HCoV-229E), and canine (CCoV) (Tresnan et al., 1996). Studies using chimeras between the human, feline, and porcine APN glycoproteins have identified two regions that are important in coronavirus receptor activity. Amino acids 717-813 of the pAPN are required for TGEV receptor activity (Delmas et al., 1994) and amino acids 670-840 of fAPN are important for receptor activity of feline and porcine viruses (Hegyi and Kolb, 1998). Furthermore, substitution of amino acids 283-290 of pAPN The Nidoviruses (Coronaviruses and Arteriviruses).

Edited by Ehud Lavi et al., Kluwer Academic/Plenum Publishers, 2001. 
with the corresponding amino acids 288-295 of hAPN resulted in a protein that serves as a receptor for both TGEV and HCoV-229E (Kolb et al., 1997).

We used wild type and nine mutant hAPN proteins expressed in mammalian cell lines to demonstrate that the addition of a glycosylation signal in hAPN at N291 blocks HCoV-229E receptor activity.

\section{MATERIALS AND METHODS}

IFA assays were done as described (Tresnan et al., 1996) using MAbs specific for hAPN (WM47) or HCoV-229E spike glycoprotein (5-11H.6). BHK-21 cells were transfected using lipofectamine ${ }^{\mathrm{TM}}$ (GIBCO Laboratories) according to manufacturer's instructions. hAPN expression plasmids were mutated using primers encoding the desired nucleotide changes and PFU polymerase was used to extend the primers. This was followed by DpnI digestion and transformation of E. coli DH5 $\alpha$ (GIBCO Laboratories). Clones were screened by restriction digestion and sequenced to verify the desired mutations.

\section{RESULTS}

\subsection{Substitution of hAPN amino acids $288-295$ for the corresponding pAPN sequence blocked infection by HCoV-229E}

BHK-21 cells were transfected with plasmids that express wild type hAPN (wt-hAPN) or a mutant hAPN containing nucleotide changes that encode 6 amino acid changes to the corresponding pAPN amino acids at 288, 289, 291, 292, 293, and 295 (p6-hAPN). Two days post-transfection the cells have similar levels of APN expression as determined by IFA with anti hAPN MAb WM47 (Table 1). Transfected cells were inoculated with $\mathrm{HCoV}-229 \mathrm{E}$ and $20 \mathrm{~h}$. later the receptor activity was determined by IFA using anti spike MAb 5-11H.6. Cells expressing wt-hAPN are infected by $\mathrm{HCoV}-229 \mathrm{E}$. In contrast, cells expressing p6-hAPN and those transfected by the empty expression plasmid (pCi-neo) are not infected by HCoV-229E (Table 1). The 6 amino acids from pAPN used to create p6-hAPN had a putative N-linked glycosylation site. Reversion of this site by mutagenesis of p6-hAPN to change asparagine 291 to glutamic acid (N291E) or threonine 293 to glutamine (T293Q), generated p6-hAPN/N291E and p6hAPN/T293Q, respectively. Transfection of BHK-21 cells with wt-hAPN, 
p6-hAPN, p6-hAPN/N291E, p6-hAPN/T293Q or pCi-neo resulted in expression of hAPN in all of the cells except those transfected by empty vector as demonstrated by IFA (Table 1). HCoV-229E inoculation of these transfected cells resulted in infection of cells expressing wt-hAPN or the revertants (p6-hAPN/N291E and p6-hAPN/T293Q), but cells transfected with p6-hAPN or pCi-neo remained nonpermissive (Table 1). None of these APN expression constructs have receptor activity for the porcine coronavirus TGEV (Table 1).

\subsection{Glycosylation of hAPN at amino acid 291 blocked infection by $\mathrm{HCoV}-229 \mathrm{E}$}

We used mutagenesis to insert a glycosylation signal at N291 of wthAPN to determine if glycosylation here was the only change needed to block HCoV-229E infection. The wt-hAPN expression plasmid was mutated at E291N and Q293T creating hAPN/N291KT. Then hAPN/N291KT was reverted to wild type at N291E or T293Q creating two other expression constructs, hAPN/E291KT and hAPN/N291KQ. Transfection of BHK-21 cells with wt-hAPN and each mutant hAPN expression plasmid resulted in hAPN expression as evidenced by IFA with MAb WM47 $48 \mathrm{~h}$. post-transfection. IFA of the transfected BHK-21 cells with anti-S glycoprotein MAb (5-11H.6) 20 h. p.i. with HCoV-229E illustrated that cells expressing wt-hAPN, hAPN/E291KT or hAPN/N291KQ were HCoV-229E permissive (Table 1). Cells transfected with hAPN/N291KT, which has the glycosylation signal, have markedly fewer IFA positive cells (Table 1).

Table 1.

\begin{tabular}{|c|c|c|c|c|c|}
\hline APN & $\begin{array}{c}\text { Amino Acids } \\
288-295 \\
\end{array}$ & $\begin{array}{c}\text { hAPN } \\
\text { Expression }\end{array}$ & $\begin{array}{c}\text { Glycosylation } \\
\text { of N291 }\end{array}$ & $\begin{array}{c}\text { HCoV-229E } \\
\text { Permissive }\end{array}$ & $\begin{array}{c}\text { TGEV } \\
\text { Permissive }\end{array}$ \\
\hline wt-hAPN & DYVEKQAS & $Y$ & $\mathrm{~N}$ & $Y$ & $\mathrm{~N}$ \\
\hline PAPN & QSVNETAQ* & $\mathrm{NA}$ & $Y$ & $\mathrm{~N}$ & $Y$ \\
\hline p6-hAPN & QSVNETAQ & $\mathrm{Y}$ & Y & $\mathrm{N}$ & $\mathrm{N}$ \\
\hline p6-hAPN/N291E & QSVEETAQ & $Y$ & $\mathrm{~N}$ & Y & $\mathrm{N}$ \\
\hline p6-hAPN/T293Q & QSVNEQAQ & $Y$ & $\mathrm{~N}$ & Y & $\mathrm{N}$ \\
\hline hAPN/N291KT & DYVNKTAS & Y & Y & $\mathrm{N}$ & ND \\
\hline hAPN/E291KT & DYVEKTAS & $Y$ & $\mathrm{~N}$ & $Y$ & ND \\
\hline $\mathrm{hAPN} / \mathrm{N} 291 \mathrm{KQ}$ & DYVNKQAS & $\mathrm{Y}$ & $\mathrm{N}$ & $Y$ & ND \\
\hline pCi-neo & & NA & NA & $\mathrm{N}$ & $\mathrm{N}$ \\
\hline
\end{tabular}

Amino acids are listed in single letter code, ${ }^{*}$ indicates amino acids 283-290 in pAPN, $\mathrm{Y}=$ yes, $\mathrm{N}=$ no, $\mathrm{NA}=$ not applicable and $\mathrm{ND}=$ not determined 


\subsection{Removal of a glycosylation signal at amino acid 818 of hAPN does not make hAPN a receptor for TGEV or FECoV}

Feline APN has receptor activity for feline, canine, human and porcine coronaviruses. The fAPN has fewer predicted glycosylation sites than hAPN or pAPN. We found a potential N-linked glycosylation site in hAPN at amino acids $818-820$ that is not in the analogous regions of pAPN or fAPN. To determine if the removal of this glycosylation signal converted hAPN into a functional receptor for porcine or feline coronaviruses, we removed it from wt-hAPN and p6-hAPN by creating hAPN/N818E, p6-hAPN/N818E and $\mathrm{p} 6 \mathrm{-hAPN} / \mathrm{T} 820 \mathrm{E}$. These plasmids were transfected into nonpermissive CMT93 cells. These cells were selected for G418 resistance and sorted for similar hAPN expression levels by FACS with MAb WM47. These stably transfected CMT93 cell lines and Felis catus cell whole fetus (FCWF) cells were inoculated with HCoV-229E, TGEV or FECoV (79-1683). The FCWF cells were used as a positive control because they express fAPN and are permissive to $\mathrm{HCoV}-229 \mathrm{E}, \mathrm{TGEV}$, and $\mathrm{FCoV}$ (Tresnan et al., 1996). Immunobloting of proteins isolated $20 \mathrm{~h}$. p.i. showed that removal of the potential glycosylation site at N818 of hAPN did not alter HCoV-229E receptor activity (Table 2). Although hAPN/N818E, like the analogous region of fAPN, lacks an N-linked glycosylation signal at amino acid 818, it lacked receptor activity for TGEV and FCoV (Table 2). Removal of the 818-820 glycosylation signal from p6hAPN yielded a protein predicted to be glycosylated at N291 and lack the signal at amino acid 818 which is similar to pAPN. Cells expressing proteins from p6-hAPN/N818E or p6hAPN/T820E lacked receptor activity for TGEV and FECoV (Table 2).

Table 2.

\begin{tabular}{lccccc}
\hline & \multicolumn{2}{c}{ Glycosylation } & \multicolumn{3}{c}{ Permissive For } \\
APN & N291 & N818 & HCoV-229E & TGEV & FECoV \\
\hline fAPN & $\mathrm{N}$ & $\mathrm{N}$ & $\mathrm{Y}$ & $\mathrm{Y}$ & $\mathrm{Y}$ \\
pAPN & $\mathrm{Y}$ & $\mathrm{N}$ & $\mathrm{N}$ & $\mathrm{Y}$ & $\mathrm{N}$ \\
wt-hAPN & $\mathrm{N}$ & $\mathrm{Y}$ & $\mathrm{Y}$ & $\mathrm{N}$ & $\mathrm{N}$ \\
hAPN/N818E & $\mathrm{N}$ & $\mathrm{N}$ & $\mathrm{Y}$ & $\mathrm{N}$ & $\mathrm{N}$ \\
p6-hAPN & $\mathrm{Y}$ & $\mathrm{Y}$ & $\mathrm{N}$ & $\mathrm{N}$ & $\mathrm{N}$ \\
p6-hAPN/N818E & $\mathrm{Y}$ & $\mathrm{N}$ & $\mathrm{N}$ & $\mathrm{N}$ & $\mathrm{N}$ \\
p6-hAPN/T820E & $\mathrm{Y}$ & $\mathrm{N}$ & $\mathrm{N}$ & $\mathrm{N}$ & $\mathrm{N}$ \\
pCi-neo & $\mathrm{NA}$ & $\mathrm{NA}$ & $\mathrm{N}$ & $\mathrm{N}$ & $\mathrm{N}$ \\
\hline
\end{tabular}

$\mathrm{Y}=$ yes, $\mathrm{N}=$ no and $\mathrm{NA}=$ not applicable 


\section{DISCUSSION}

The species specificity and tissue tropism of viruses are, in large part, determined by the specificity of virus-receptor interactions. The p6-hAPN mutant that has pAPN amino acids 283-290 substituted for 288-295 of hAPN, completely lacked receptor activity for HCoV-229E. The most important change among these mutations was the introduction of a potential glycosylation signal from amino acids 291-293 of hAPN. To determine if the introduction of the glycan at N291 was responsible, two other mutants generated from p6-hAPN that changed N291E or T293Q but also had 5 amino acid changes in this region were tested for HCoV-229E receptor activity. These two revertants were functional $\mathrm{HCoV}-229 \mathrm{E}$ receptors. Feline APN also has amino acid differences from hAPN at D288, K292, Q293 and S295. This suggests that the block in receptor activity of p6hAPN is due to glycosylation of N291 rather than the individual amino acid substitutions. This is best demonstrated by the hAPN/N291KT construct that contained only this glycosylation signal and lacked receptor activity. However, revertants (hAPN/E291KT and hAPN/N291KQ) that lack this signal have $\mathrm{HCoV}-229 \mathrm{E}$ receptor function. This data demonstrates that the glycosylation of hAPN at N291 blocks infection.

Studies of a region of APN essential for TGEV and FECoV receptor activity identified a potential glycosylation signal at N818 of hAPN that is not conserved in pAPN or fAPN. However mutants of hAPN created to remove this potential glycosylation signal did not have receptor activity for TGEV or FECoV, but retained receptor activity for $\mathrm{HCoV}-229 \mathrm{E}$. These results show that removal of a potential glycosylation signal at N818 of hAPN is not sufficient to confer receptor activity for porcine and feline coronaviruses.

\section{ACKNOWLEDGEMENTS}

The authors would like to thank Pierre Talbot for the anti spike MAb 511H.6, Justin Hagee for his technical assistance, Karen Helm at the UCHSC Cancer Center for FACS and Dr. Bruce Zelus for his critique of this manuscript. This work was supported by NIH grant AI26075 and Dr. Wentworth was supported by NIH Neurovirology-Molecular Biology Training Grant-T32 NS07321. 


\section{REFERENCES}

Delmas, B., Gelfi, J., Kut, E., Sjostrom, H., Noren, O., and Laude, H.(1994). Determinants essential for the transmissible gastroenteritis virus-receptor interaction reside within a domain of aminopeptidase- $\mathrm{N}$ that is distinct from the enzymatic site. Journal of Virology 68, 5216-5224.

Delmas, B., Gelfi, J., L'Haridon, R., Vogel, L.K., Sjostrom, H., Noren, O., Laude, and H.(1992). Aminopeptidase $\mathrm{N}$ is a major receptor for the entero-pathogenic coronavirus TGEV. Nature 357, 417-420.

Delmas, B., Gelfi, J., Sjostrom, H., Noren, O., and Laude, H.(1993). Further characterization of aminopeptidase- $\mathrm{N}$ as a receptor for coronaviruses. Advances in Experimental Medicine \& Biology 342, 293-298.

Hegyi, A. and Kolb, A.F.(1998). Characterization of determinants involved in the feline infectious peritonitis virus receptor function of feline aminopeptidase N. Journal of General Virology 79, 1387-1391.

Kenny, A.J. and Maroux, S.(1982). Topology of microvillar membrance hydrolases of kidney and intestine. [Review] [153 refs]. Physiological Reviews 62, 91-128.

Kolb, A.F., Hegyi, A., and Siddell, S.G.(1997). Identification of residues critical for the human coronavirus $229 \mathrm{E}$ receptor function of human aminopeptidase N. Journal of General Virology 78, 2795-2802.

Lachance, C., Arbour, N., Cashman, N.R., and Talbot, P.J.(1998). Involvement of aminopeptidase $\mathrm{N}(\mathrm{CD} 13)$ in infection of human neural cells by human coronavirus $229 \mathrm{E}$. Journal of Virology 72, 6511-6519.

Look, A.T., Ashmun, R.A., Shapiro, L.H., and Peiper, S.C.(1989). Human myeloid plasma membrane glycoprotein CD13 (gp150) is identical to aminopeptidase N. Journal of Clinical Investigation 83, 1299-1307.

Noren, O., Sjostrom, H., and Olsen, J.(1997). Cell-Surface Peptidases in Health and Disease (Kenney, A.J. and Boustead, C.M., Eds.) BIOS Scientific Publishers, Oxford. 175-191.

Riemann, D., Kehlen, A., and Langner, J.(1999). CD13 - not just a marker in leukemia typing [Review]. Immunology Today 20, 83-88.

Tresnan, D.B., Levis, R., and Holmes, K.V.(1996). Feline aminopeptidase N serves as a receptor for feline, canine, porcine, and human coronaviruses in serogroup I. Journal of Virology 70, 8669-8674.

Yeager, C.L., Ashmun, R.A., Williams, R.K., Cardellichio, C.B., Shapiro, L.H., Look, AT, and Holmes, K.V.(1992). Human aminopeptidase $\mathrm{N}$ is a receptor for human coronavirus 229E. Nature 357, 420-422. 